\title{
Torsional Oscillation Analysis of a Hybrid Electric Bus's Parking Condition
}

\author{
Xiaoguang Guo and Hongwen He \\ National Engineering Laboratory for Electric Vehicles, Department of Mechanical and Vehicular Engineering, Beijing Institute of Technology, \\ Beijing100081, China
}

\begin{abstract}
The aim of this study is to solve the problem of torsional oscillation in the parking condition of a hybrid electric bus. The dynamic amplification factor formula is derived, and its main optimization variables is found by the method of sensitivity analysis. The maximum torque equation of the hybrid electric bus is constructed and optimized, and the optimization results is verified by experiment finally.
\end{abstract}

\section{Introduction}

Compared with the internal combustion engine bus, the structure of hybrid electric bus's transmission system is more complex. Both of the transmission system constitution's changing and the rapid vary of the motor torque put forward new challenges in the hybrid electric bus's shafting systems [1], so the torsional oscillation is the main problem that the transmission system needs to face.

In the parking condition, a kind of hybrid electric bus's engine is killed by the torque from the ISG motor, midway through spline shaft and torsion shock absorber. When the torsional frequency of the ISG motor and the natural frequency of the transmission system is the same or similar, the resonance phenomenon will be produced [2]-[5]. When this starts to happen, the torque value of the transmission system should be much greater than the torque provided by the ISG motor, and the shaft may be broken off [6].

\section{Dynamic amplification factor}

The structure of a hybrid electric bus transmission system is shown in Fig. 1. The transmission system is composed of the engine, the torsion shock absorber, the spline shaft, the ISG motor.

In the parking condition, torque provided by ISG motor passes through the spline shaft and torsional shock absorber to the engine starts. In this process, the ISG motor is the driving source, and the spline shaft, the torsion shock absorber, the engine is the vibration's receiving terminal.

In order to express convenience, the spline shaft, torsional shock absorber, engine components as part one, and the ISG motor is treated as part two. When resonances occurred in transmission systems, the natural frequency of part one is the same or similar as part two.

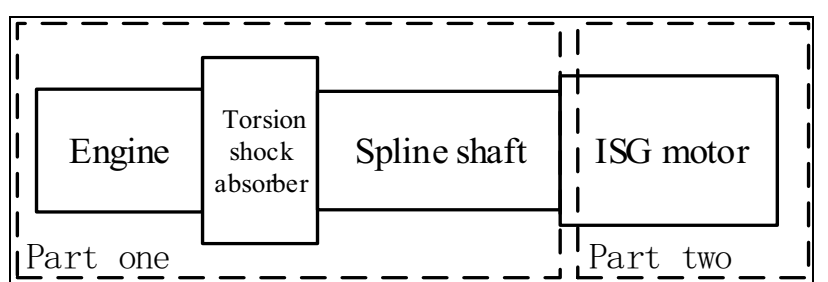

Figure 1. Schematic diagram of hybrid electric car's transmission system

\subsection{Nature frequency}

The mechanical model of Fig. 1 is shown in Fig. 2.

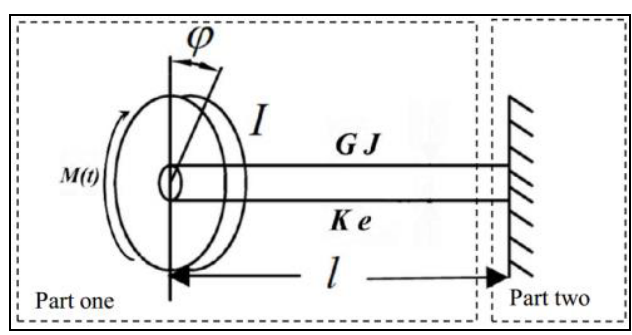

Figure 2. Mechanical model of hybrid electric car's transmission system

Set the equivalent length of the part one is $l$, the equivalent shear modulus is $G$, the inertia moment of the equivalent section is $J$, then the torsional stiffness $K$ and the flexibility $e$ is as follow:

$$
K=\frac{G J}{l}, e=\frac{l}{G J}
$$

Set the angular displacement of any instantaneous part one is $\phi$, then the elastic moment is: 


$$
U=-K \phi
$$

The negative sign in this equation means the direction of elastic moment is always opposite to the angular displacement $\phi$.

According to D'Alembert principle, the differential equations of motion is constructed as follow [7]:

$$
S=-I \ddot{\phi}
$$

The inertia moment and elastic moment are balanced, so we may get the equation:

$$
S+U=0, \text { thus : } I \ddot{\phi}+\frac{1}{e} \phi=0
$$

The solution of the differential equation is the equation of part one:

$$
\phi=A \sin (p t+\varepsilon)
$$

which $: p=\sqrt{\frac{1}{I e}}=\sqrt{\frac{G J_{p}}{I l}}$

In formula (6) $p$ is part one's natural frequency expression.

\subsection{Dynamic amplification factor}

The dynamic amplification factor here means the ratio of toque on part one and toque issued by ISG motor.

In formula (4), the torque of ISG motor's expression is as follow:

$$
T=M \sin \omega t
$$

Set damping moment as:

$$
R=-c \dot{\phi}
$$

thus,

$$
I \ddot{\phi}+c \dot{\phi}+\frac{1}{e} \phi=M \sin \omega t
$$

Deforming formula (9) as:

$$
\ddot{\phi}+2 \mathrm{n} \dot{\phi}+p^{2} \phi=h \sin \omega t
$$

which: $n=\frac{c}{2 I}$

The general solution for differential formula (10) is:

$$
\phi=A e^{-n t} \sin \left(p_{d} t+\varepsilon\right)+A_{1} \sin (\omega t-\psi)
$$

Solving differential equations (10), and the dynamic amplification factor will be obtained as:

$$
\beta=\frac{1}{\sqrt{\left[1-\left(\frac{\omega^{2}}{p^{2}}\right)\right]^{2}+4\left(\frac{n^{2}}{p^{2}}\right)\left(\frac{\omega^{2}}{p^{2}}\right)}}
$$

Getting formula (6) and (11) into formula (13), we may safely get the following results:

$$
\beta=\frac{1}{\sqrt{\left(1-\omega^{2} \frac{I l}{G J_{p}}\right)^{2}+\left(\frac{c}{I}\right)^{2} \omega^{2}\left(\frac{I l}{G J_{p}}\right)^{2}}}
$$

\section{Sensitivity analysis}

\subsection{The design of sensitivity analysis}

The independent variable is used to scatter sample points in design space, so as to constructing the mathematical expression of the independent variable and the objective function, and then the construction of pareto chart between the variable and the objective function will be completed, thus the sensitivity analysis will be finished.

As the upper and lower limit of the independent variable is very wide, and in order to cover the design space with the limited number of sampling points, the construction of sample points is completed by Latin hypercube method. Latin hypercube method is a statistical method for generating a sample of plausible collections of parameter values from a multidimensional distribution.

In the context of statistical sampling, a square grid containing sample positions is a Latin square if (and only if) there is only one sample in each row and each column $^{[8]}$. A Latin hypercube is the generalization of this concept to an arbitrary number of dimensions, whereby each sample is the only one in each axisaligned hyperplane containing it. Fig. 3 direct comparison the difference between Latin hypercube method and full factorial method.

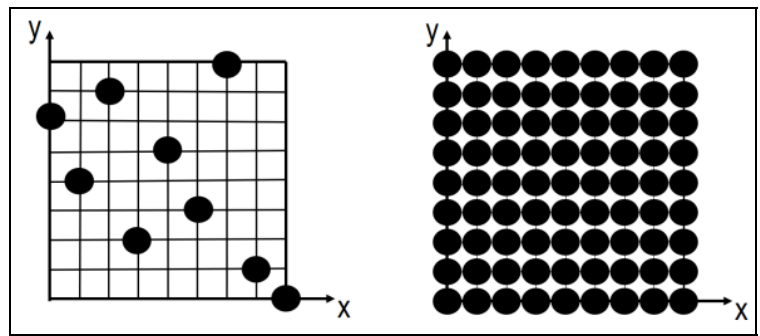

Figure 3. Latin hypercube method(left) full factorial method(right)

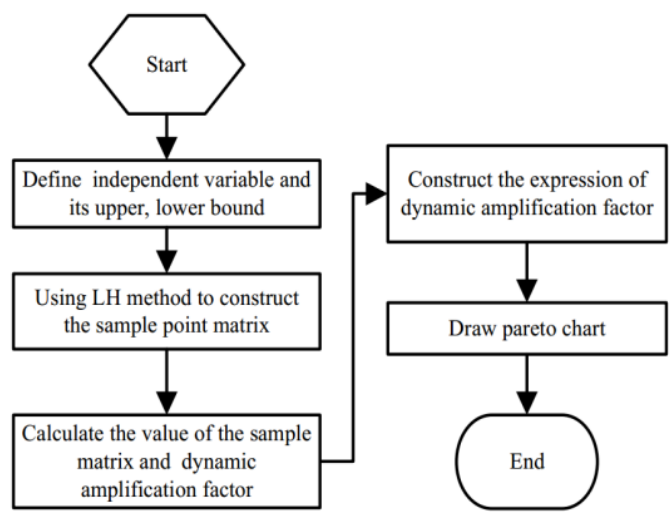

Figure 4. Sensitivity analysis flow chart for 


\section{transmission system}

Fig. 4 shows the specific processes of the sensitivity analysis for transmission system.

\subsection{Sensitivity analysis results}

According to Fig. 4, formula (14) should be expressed as:

$$
\begin{aligned}
\beta= & 10.62 \omega-10.58 G J+8.08 G I \\
& +6.68 G \omega-6.31 L \omega+6.24 I J+5.54 G L \\
& +4.68 J^{2}-4.57 c G-4.53 I^{2}+\ldots
\end{aligned}
$$

Using formula (15) to draw the pareto chart of each independent variable's dynamic magnification factor, and the results shows in Fig. 5.

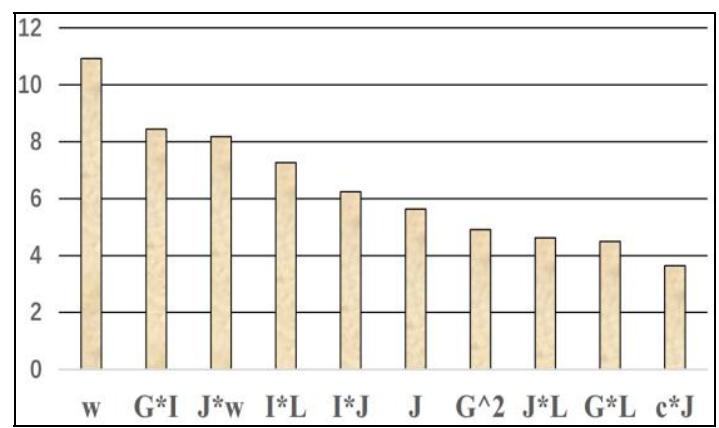

Figure 5. Sensitivity analysis of transmission system - Pareto chart

From Fig. 5, we may safely find out that the independent variable of $\omega$ is the highest sensitivity to dynamic amplification factor. The second highest sensitivity to dynamic amplification factor is the product of the shear modulus $\boldsymbol{G}$ and the moment of inertia $\boldsymbol{I}$. The third one is the inertia moment of the equivalent section $J$ and $\omega$.

\section{Torque optimization}

\subsection{Independent variables}

Generally speaking, the design variables are the greatest impact on the sensitivity of the target function. Considering the sensitivity analysis's result, the actual engineering structure design, parts processing and other factors, the shear modulus of elasticity $\boldsymbol{G}$ is chosen as the independent variable.

\subsection{Constraint condition}

In order to make the transmission system work normally in all the working conditions, the maximum torque of the shaft section is less than the allowable value, that is:

$$
|T| \leq \mathrm{T}^{*}
$$

$|T|$ is the representative of the actual absolute torque, and $\mathrm{T}^{*}$ is the representative of the allowable stress.

\subsection{Objective function}

Minimization of each axis section's torque $T$ is chosen as the objective function, which $T=T_{I S G} \beta$.In order to ensure the hybrid electric bus in the parking condition does not occur the torsion form of resonance, it is need to iterates over the bus's frequency in the parking condition. It is need to traverse frequency $0 \sim 50 \mathrm{~Hz}$, and the step size is 0.1 .

The torque optimization equation of the transmission system can be described as:

$$
\left\{\begin{array}{l}
\text { loop } i=0,0.1,50 \\
\text { find } G \\
\min \mathrm{T}_{i}(\mathrm{G})=\mathrm{T}_{I S G i} \beta_{i} \\
\text { s.t. } \max \left\{\mathrm{T}_{i}(\mathrm{G})\right\} \leq \mathrm{T}^{*} \\
G^{L} \leq G \leq G^{U}
\end{array}\right.
$$

\subsection{Selection algorithm}

To prevent the optimization into local optimization and couldn't find global optimal value, here the algorithm of genetic algorithm(GA) is chosen.

\subsection{The flow chart of optimization}

The flow chart of the torque optimization is shown in Fig. 6.

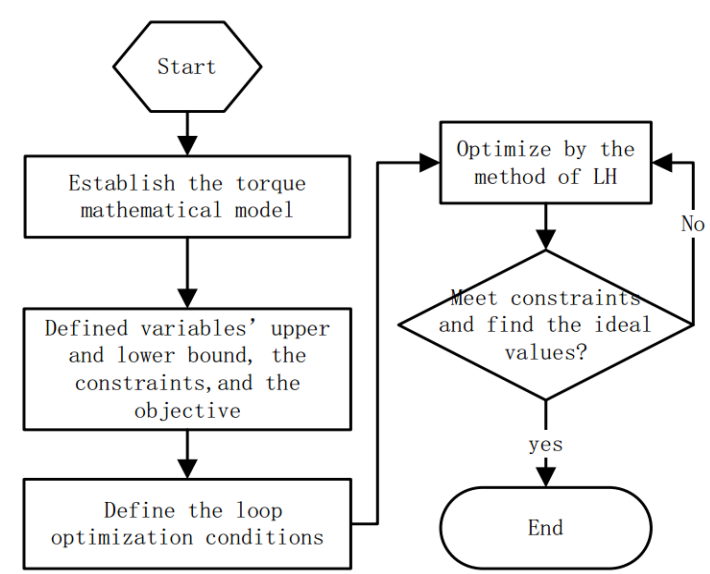

Figure 6. The flow chart of torque optimization for transmission system

\subsection{Optimization result analysis}

Using the GA algorithm, the optimal value is obtained after 224 iteration calculation, and the maximum torque history curve of the transmission system is shown in Fig. 7.

Before optimization, in the hybrid electric bus's parking condition, the ISG motor and transmission system has occurred the torsional resonance, and the maximum torque of the transmission system is $1520 \mathrm{Nm}$, which beyond the allowable stress $1200 \mathrm{Nm}$ and it is in the danger stage. 
After optimization, the maximum torque of transmission system is $1100 \mathrm{Nm}$, which has been down by $27.6 \%$, and the result is also in the range of safety torque.

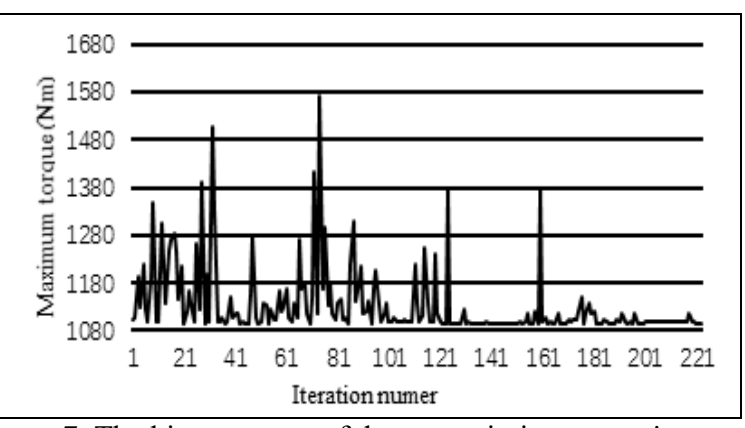

Figure 7. The history curve of the transmission system's maximum torque

\section{Experimental verification of the parking condition}

The experimental platform of the hybrid electric bus transmission system is built, as shown in Fig. 8.

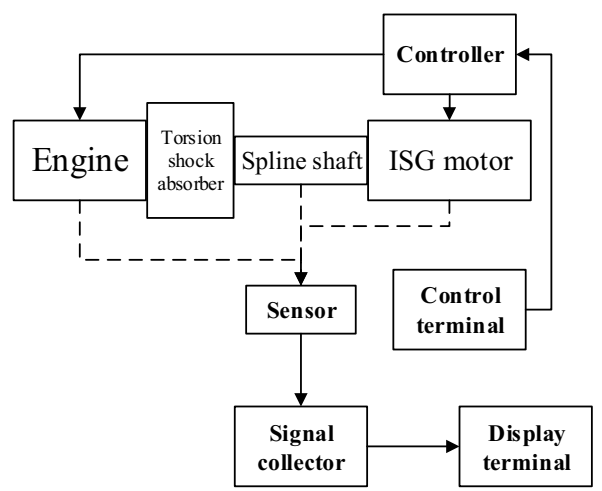

Figure 8. Schematic diagram of experimental platform of transmission system

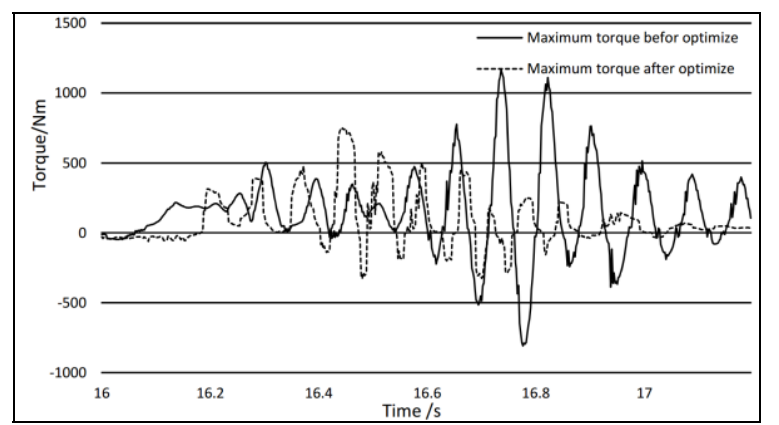

Figure 9. Time domain diagram of the maximum torque before and after the optimization

The transmission system's shear modulus of elasticity $\boldsymbol{G}$ is changed according to the optimization results, and Fig. 9 shows the maximum torque of the drive system. We can obtain that, after optimization, the maximum torque of the drive system is reduced to $749.01 \mathrm{Nm}$.

The maximum torque of the hybrid electric bus transmission system is reduced by the way of shifting frequency, and this means the hybrid electric bus is also provided a more reliable security.

\section{Conclusions}

This paper takes hybrid electric bus's transmission system as the research object. The expression of the dynamic amplification factor for the transmission system is deduced, and the sensitivity analysis is carried out. The maximum torque equations of the parking condition for the hybrid electric bus is constructed and optimized.

The main conclusions is as follows:

1) By the sensitivity analysis of the dynamic amplification factor expression, the transmission system of shear modulus of elasticity $G$, moment of inertia $J$, rotational inertia $\boldsymbol{I}$ are coupling.

2) In order to solve the problem of the torsional oscillation in hybrid electric bus, using the method of sensitivity analysis to find out the maximum sensitivity of the parameter in the expression of dynamic amplification factor, and making this parameter as independent variable to reduce the torque of the optimization process is feasible. These work will provide a set of guidelines for the issue of the torsional oscillation in the future.

\section{References}

1. Kim H, Kim J, Lee H, et al. Mode Transition Control Using Disturbance Compensation for a Parallel Hybrid Electric Vehicle[J]. Proceedings of the Institution of Mechanical Engineers Part D Journal of Automobile Engineering, 225(225):150-166, (2011).

2. Walker P D, Zhang N. Modelling of dual clutch transmission equipped powertrains for shift transient simulations[J]. Mechanism \& Machine Theory, 60(1):47-59, (2013).

3. Zhang J, Tang X, Yu H, et al. Multi-body dynamics and noise analysis for the torsional vibration of a power-split hybrid driveline[J]. Proceedings of the Institution of Mechanical Engineers, Part K: Journal of Multi-body Dynamics, 228(4):366-379, (2014).

4. Iman, R.L.; Helton, J.C.; and Campbell, J.E.. "An approach to sensitivity analysis of computer models, Part 1. Introduction, input variable selection and preliminary variable assessment". Journal of Quality Technology 13(3): 174-183, (1981).

5. Iman, R.L.; Davenport, J.M.; Zeigler, D.K.. Latin hypercube sampling (program user's guide), (1980).

6. Mckay M D, Conover R J B W J. A Comparison of Three Methods for Selecting Values of Input Variables in the Analysis of Output From a Computer Code[J]. Technometrics, 42(1):55-61, (2012).

7. Eglajs, V.; Audze P.. "New approach to the design of multifactor experiments".Problems of Dynamics and Strengths. 35 (in Russian) (Riga: Zinatne Publishing House):104-107, (1977).

8. Iman, R.L.; Helton, J.C.; Campbell, J.E.. "An approach to sensitivity analysis of computer models, Part 1. Introduction, input variable selection and preliminary variable assessment". Journal of Quality Technology 13 (3): 174-183, (1981). 